\title{
Effect of mandibular complete dentures relining on occlusal force distribution using T-scan system
}

\author{
Eman M. Ibraheem ${ }^{1}$ and Hisham S. ElGabry ${ }^{2^{*} \text { (D) }}$
}

\begin{abstract}
Background: This study aimed to evaluate the effect of mandibular complete dentures relining using soft relining material on the distribution of various occlusal forces using T-Scan system. Fifty completely edentulous patients having their conventional complete dentures earlier fabricated and utilized were selected for this study. Patients were controlled diabetics, characterized by having their residual alveolar ridges moderately developed and lined with firm mucoperiosteum. Mandibular complete dentures were relined with soft denture liner and T-Scan device was used for occlusal force distribution measurement prior to denture relining and three months thereafter the relinning procedure.
\end{abstract}

Results: Comparison between occlusal forces percentages before and after denture relining revealed that occlusal forces percentages was significantly lower after denture relining in anterior area, significantly higher after denture relining in right posterior area, where it was insignificantly higher after relining in left posterior area.

Conclusions: Our findings revealed that the use of soft denture liner for mandibular complete denture relining significantly improved the occlusal load distribution.

Clinical trial registration Trial registration NCT, NCT04701970. Registered 23/11/2020-Retrospectively registered, https://clinicaltrials.gov/ct2/show/NCT04701970

Keywords: Complete dentures relining, Occlusal force distribution, T-scan system

\section{Background:}

Alveolar bone reduction is almost always a consequent of teeth loss associated with advanced age. As a matter of fact, the reduced ridge height and width greatly affects complete dentures retention and stability specially the mandibular complete denture (Misch 2008; Ibraheem et al. 2019; Doundoulakis et al. 2003). Furthermore, reduced lower denture retention and stability has a great impact on the patients' masticatory efficiency, self-confidence, prosthesis usage satisfaction, and, hence, their

\footnotetext{
*Correspondence: Hishamelgabry@live.com

2 Prosthodontics Department, National Research Centre-MSA University, Cairo, Egypt

Full list of author information is available at the end of the article
}

quality of life (Jain and Rathee 2019; Ibraheem and Ham$\operatorname{mad} 2019)$.

The mandibular complete denture retention could be more superior by applying different methods as clinical guidelines, flexible materials, pre-prosthetic surgeries that improve denture foundation or by using dental implants (Ibraheem et al. 2019; Pradeep et al. 2015). Nevertheless, soft denture liners and denture adhesives are other modalities that could improve complete denture retention (Ibraheem and Hammad 2019; Pradeep et al. 2015; Elmorsy et al. 2015). Denture lining materials whether temporary or permanent are highly effective materials that greatly improve denture adaptation to underlying tissues. Hard liners are mainly resin-based polymers which are manufactured from poly-methyl 
methacrylate (PMMA) (Atay et al. 2012). Whereas, Soft resilient liners whether long-term or short-term types are plasticized acrylic resins or silicone elastomeric type of materials which can equally distribute functional load on denture foundation areas (Atay et al. 2012; Kreve and Dos Reis 2019).

It is to be mentioned that complete denture retention is, in part, influenced by denture occlusion. Most denture wearers consciously perform random, occlusal contact movements at all times. These contacts may result from functional activity (swallowing) or parafunction (bruxism or clenching). A well balanced denture occlusion is intended to minimize the adverse effects of functional and parafunctional movements by widely distributing these forces to the denture bearing areas. Therefore, a properly balanced denture occlusion may serve to properly distribute occlusal forces that may act to disturb denture stability (Kreve and Dos Reis 2019; Koos et al. 2010).

Conventional clinical assessment techniques for occlusal contacts equilibration include the use of either articulating paper/indicator wax or patient feedback (Koos et al. 2010; Qadeer et al. 2012). Whereas, even though articulating paper is the most commonly used method, it is an undependable indicator of actual occlusal load as its markings depend mainly on the occlusion quality, anatomy, and type of saliva, which can cause false positive marks (Kerstein 2008).

Lately, the occlusal equilibration using computerized assessment methods as the T-Scan system that appeared in 1987 has been widely used and offers reliable results. The latest version (T-Scan III) provides a unique method of occlusal load evaluation dynamically from the time of initial tooth contact to maximum teeth intercuspation. This system records different values for occlusal force besides, allowing occlusal balance evaluation through recording values for occlusion and dis-occlusion numerically (Maness et al. 1987; Martins et al. 2014; Kerstein et al. 2013).

Occlusion time is known as "time from the first occlusal contact to maximum teeth intercuspation", whereas the disocclusion time is known as "time from maximum teeth intercuspation to complete disocclusion" (Gümüs et al. 2013). The main target is to reach occlusion time in less than $0.2 \mathrm{~s}$ and disocclusion time in less than $0.4 \mathrm{~s}$. Less Occlusal time means less prematurity and is an indication of simultaneous occlusal contact. Whereas, the less the disocclusion time the less the stresses upon the temporomandibular joints (Kerstein and Radke 20062).

Furthermore, even distribution of occlusal forces over denture foundation areas, reduces the occlusal stress concentration and lowers possible residual ridge resorption rate. T-Scan is definitely helpful in providing data about measurable occlusal force distribution which is nearly $50 \%$ occlusal force balance on both jaw sides improving the dentures tissue adaptation during patient mastication. Thus, T-Scan usage would be of great help to achieve simultaneous occlusal contact and correct occlusal prematurities more accurately (Kerstein and Grundset 2001; Tallgren et al. 1992).

The contact areas registered by T-Scan are represented as multiple columns of different heights and colors indicating the occlusal forces intensity. The amount of occlusal force is color coded as the red color represents the maximum force and the blue color indicates the minimum force. T-Scan measures the relative occlusal force values from initial tooth contact to maximum teeth intercuspation. Furthermore, it marks the abnormal occlusal forces in every tooth in the denture which may lead to trauma or pain and thereby, helps to balance the forces on both sides of the arch (Maruo et al. 2010).

Diabetes mellitus is a chronic metabolic endocrinal disorder and is one of the most widely spread diseases which is almost found in most elderly dental patients. It has been classified into type 1, type 2, Hybrid forms, unclassified diabetes where, Type 2 accounts for more than $90 \%$ of diabetic patients (Metwally 20199; Rahman 2013).

Ketone breath, gingival and periodontal disease, alveolar ridge resorption, candidiasis, and xerostomia are the most common oral manifestations of diabetes (MauriObradors et al. 2019). Also, reduced oral mucosa resiliency is evident, which is of utmost necessity for good dentures adaptation, as complete dentures retention for diabetic patients was likely found to be less than nondiabetic patients (Mauri-Obradors et al. 2017; Kansal and Goyal 2013).

This study aimed to accurately assess the impact of soft denture liners using T-Scan III computerized occlusal analysis system on occlusal equilibration in diabetic complete denture patients.

\section{Methods}

The crossover clinical study was made using already constructed and utilized complete dentures before attempting relinning procedure using soft denture liners and then after being relined with soft denture liners. The study sample included 50 completely edentulous male patients, 49-63 years old (with an average of 56 years). Patients were chosen from the out-patient dental clinics of Medical Excellence Centre, NRC. Patients were controlled type 2 diabetes (i.e. fasting serum glucose level was higher than $126 \mathrm{mg} / \mathrm{dl}$ ) as well as the glycosylated hemoglobin level (HbA1c) does not exceed 7.5\% during the study phase. All patients were non-smokers with Angle class I classification and no history of temporomandibular/neuromuscular disorders, where bruxers and heavy clenchers were totally excluded from the study. All 
patients were characterized by having moderately developed alveolar ridges lined with firm mucoperiosteum. On the other hand, patients with medical problems or chronic diseases except diabetes or any intraoral soft or hard tissue pathology were totally excluded from the study. Intra/extra-oral examinations, panoramic radiograph and diagnostic casts were made for all selected patients. Furthermore, all patients were instructed to keep the denture fitting surfaces cleaned and get their dentures removed at bed time, keep them in a water basin overnight and massage their oral tissues gently.

\section{Prosthodontic management}

All selected patients have been daily using their conventional heat cured acrylic resin complete dentures for at least two years with definite history of mandibular dentures problems primarily related to retention and stability (Soman and Bhatnagar 2014).

After that, the mandibular complete dentures received the necessary prosthetic adjustments to achieve optimal retention and stability together with perfection of occlusal contacts, where the first occlusal force measurements were made before the dentures being relined. T-Scan device was used for occlusal force distribution measurement prior to denture relining and three months thereafter the relinning procedure.

\section{Relining technique (direct-abrasion method)}

- Relining of mandibular dentures was made by making a relief of $1-1.5 \mathrm{~mm}$ of the denture fitting surfaces and borders to achieve borders of right-angle.

- Long-term soft lining (LTSL) material (MUCOPERN-SOFT Kettenbach GmbH and Co. Kg, Germany), manipulation was performed following manufacturer instruction.

- Afterwards; Mucopren adhesive was applied in two separate layers on the mandibular denture vestibular surface and each layer was allowed $40 \mathrm{~s}$ to dry.

- Mandibular denture-fitting surface was filled with an even thickness of LTSL using the manufacturer syringe.

- Then, mandibular denture was inserted in patients' mouth and the material was molded accordingly; while asking the patient to close gently in centric occlusion with the opposing denture.

- After complete setting of LTSL material, trimming of excess material was performed and finally dentures were inspected, finished and polished.

- Strict post-insertion denture care protocol was reassured with all patients.

- All required intraoral denture adjustments were made on the day of denture insertion and continued throughout the first week make sure that all patients were free from any possible dentures discomfort.

Patients were then asked to routinely use their relined dentures daily for at least three months then; dentures retention and stability were assessed before further recording of occlusal force distribution.

\section{Evaluation of occlusal force equilibration}

The occlusal force distribution evaluation was carried out using the T-Scan device (Tekscan Inc., South Boston, MA, USA) (Figs. 1, 2). The first record was made before denture relining and the second record was made after three months of denture relining as follows:

A sensor of the appropriate size was selected according to dentures form and arch size. Then, patients were instructed to sit upright and parallel placement of the sensor to the maxillary occlusal plane was made with the midline was marked between the central incisors. To adjust the sensor sensitivity; patients were instructed to trial bite 2-4 times before recording then, they was instructed to bite in maximal intercuspation with the sensor positioned between the upper and lower dentures and to keep biting for 1-3 $\mathrm{s}$ according to the manufacturer instructions. The procedure was repeated three times and the recorded movie was saved on the computer



Fig. 1 T-Scan device and the sensors 


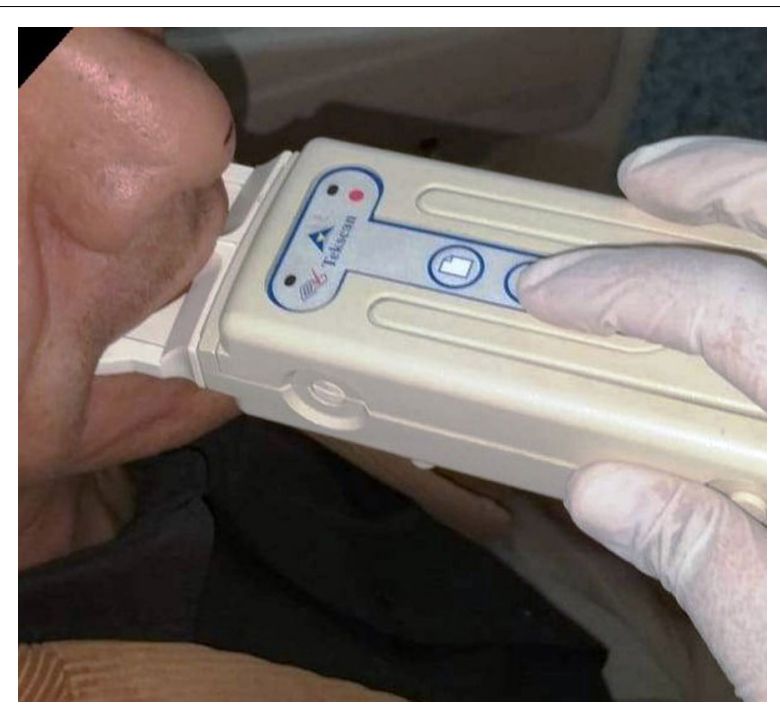

Fig. 2 T-Scan device in patient's mouth

for analysis and all measurements were made by one examiner. The percentage (\%) of occlusal force distribution for both anterior and posterior denture areas in each quadrant were recorded. Finally, the mean values were calculated from the three recordings \& the data was saved for statistical analysis (Figs. 3, 4).

\section{Results}

Data were presented as mean and standard deviation. Data were presented in one table and one graph. Statistical analysis was performed with SPSS $16{ }^{\circledR}$ (Statistical
Package for Scientific Studies), Graph pad prism and windows excel.

Paired t-test was performed between occlusal forces percentages before and after denture relining, while comparison between different areas was performed by One Way Repeated ANOVA, followed by Tukey's post hok test for multiple comparisons as presented in Table 1 and Fig. 5.

Comparison between occlusal forces percentages before and after denture relining performed by paired $t$ test revealed that occlusal forces percentages was significantly lower after denture relining in anterior area, and significantly higher after denture relining in right posterior area, where it was insignificantly higher after relining in left posterior area as presented in Table 1 and Fig. 5.

One Way Repetitive ANOVA was performed in each group to compare between three areas and revealed significant difference between them $(P<0.05)$, followed by Tukey's post hoc test for multiple comparisons which revealed significant difference in means with different superscript letters (anterior area and right posterioranterior area and left posterior before and after denture relining), while revealed insignificant difference in means with the same superscript letters (right and left posterior area before and after correction) as presented in Table 1.

\section{Discussion}

Patient satisfaction is directly affected by the denture stability; which is mainly influenced by presence of balanced occlusal contacts. Most commonly, at the time of insertion and post-insertion recall visits, complete denture

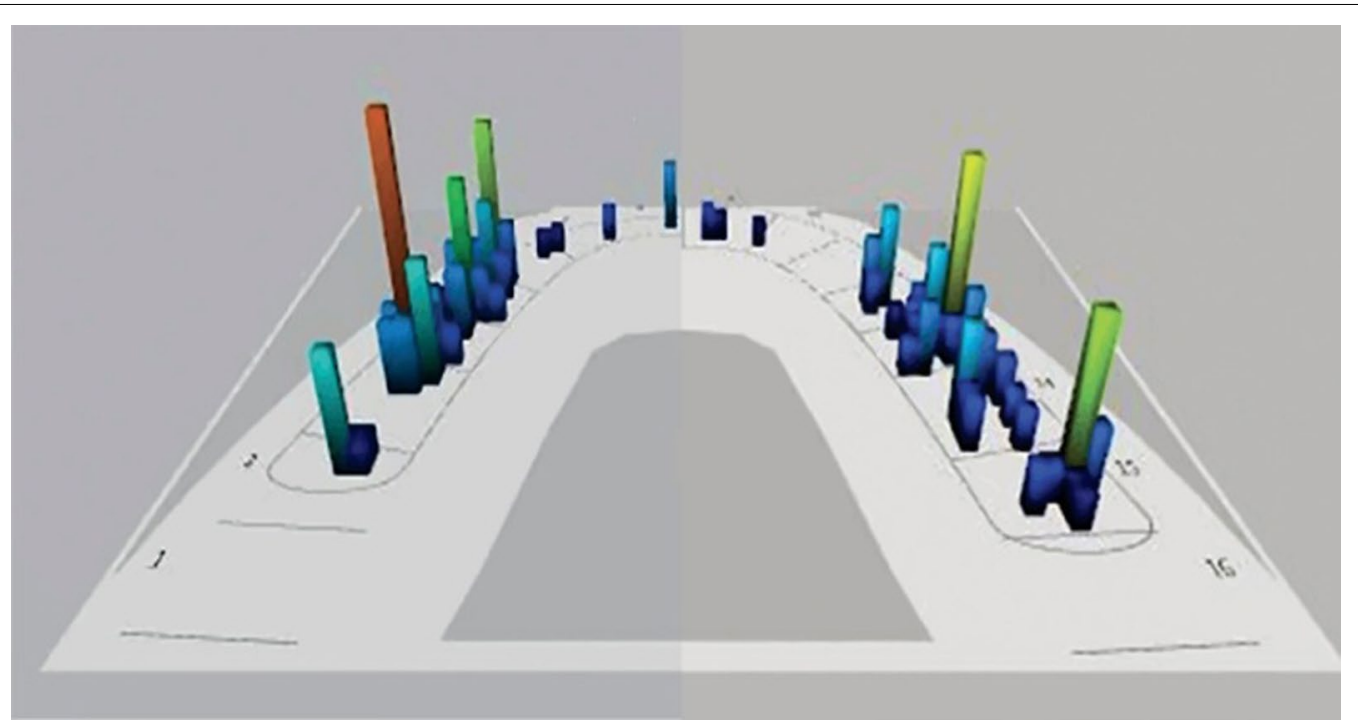

Fig. 3 T-Scan record after denture relining, the right side shows absence of high intensity force columns and more favorable force distribution pattern, while the left side shows premature contacts at particular areas, indicated by red columns (representing higher intensity forces) 




Fig. 4 T-Scan records and software analysis

Table 1 Percentages of Occlusal forces before and after denture relining in anterior, right posterior and left posterior areas

\begin{tabular}{|c|c|c|c|c|c|c|c|c|}
\hline & \multirow[t]{2}{*}{$N$} & \multicolumn{2}{|c|}{ Anterior area } & \multicolumn{2}{|c|}{ Posterior right } & \multicolumn{2}{|c|}{ Posterior left } & \multirow{2}{*}{$\begin{array}{l}P \\
\text { value }\end{array}$} \\
\hline & & $M$ & SD & $M$ & SD & $M$ & SD & \\
\hline Before relining & 50 & $15.26 \mathrm{a}$ & 4.32 & $41.98 \mathrm{~b}$ & 9.26 & $43.54 \mathrm{~b}$ & 12.14 & $0.001^{*}$ \\
\hline After relining & 50 & $9.4 \mathrm{a}$ & 0.96 & $45.24 \mathrm{~b}$ & 2.00 & $44.9 \mathrm{~b}$ & 1.73 & $0.001^{*}$ \\
\hline$P$ value & & $0.001^{*}$ & & $0.01^{*}$ & & 0.4 & & \\
\hline
\end{tabular}

$M$ mean, SD Standard deviation, $N$ count, $P$ Probability level, *Significantly different $(P<0.05)$

Means with different superscript letters were significantly different

Means with the same superscript letters were insignificantly different

occlusion is adjusted using articulating paper till reaching acceptable simultaneous occlusal contacts. However, this procedure is not accurately reliable for the study of occlusal forces distribution as the accurate analysis that combines time resolution and forces distribution within patient occlusion is not applicable when traditional methods of occlusal assessment are being used (Maruo et al. 2010).

Recently, digital occlusal analysis using T-scan device had been primarily used to evaluate various occlusion parameters. One of the main parameters is the complete dentures occlusal force distribution patterns as it can detect and locate premature contacts and occlusal interference respectively. In this study T-scan had been utilized to evaluate the occlusal force distribution percentage in conventional complete dentures before and after accurate relining procedure.
It is well known that soft liners distribute the occlusal forces uniformly across the denture-bearing area during function leading to limited local pressure points, improved occlusal contact and premature contact point's reduction that usually results from denture movement during function (Rani et al. 2017).

To ensure valid and reliable results, exclusion of interexaminer variations was insured by making all of the T-scan recordings by the same single examiner who also assessed denture retention and stability. Similarly, only one clinician performed all occlusal adjustments and occlusal parameter recordings for more accurate results (Ibraheem and Hammd 2020).

Regarding anterior area, the study results had revealed that T-scan analysis used for evaluating the percentage of force distribution and for determination of unseen premature contacts that could not be accurately detected 


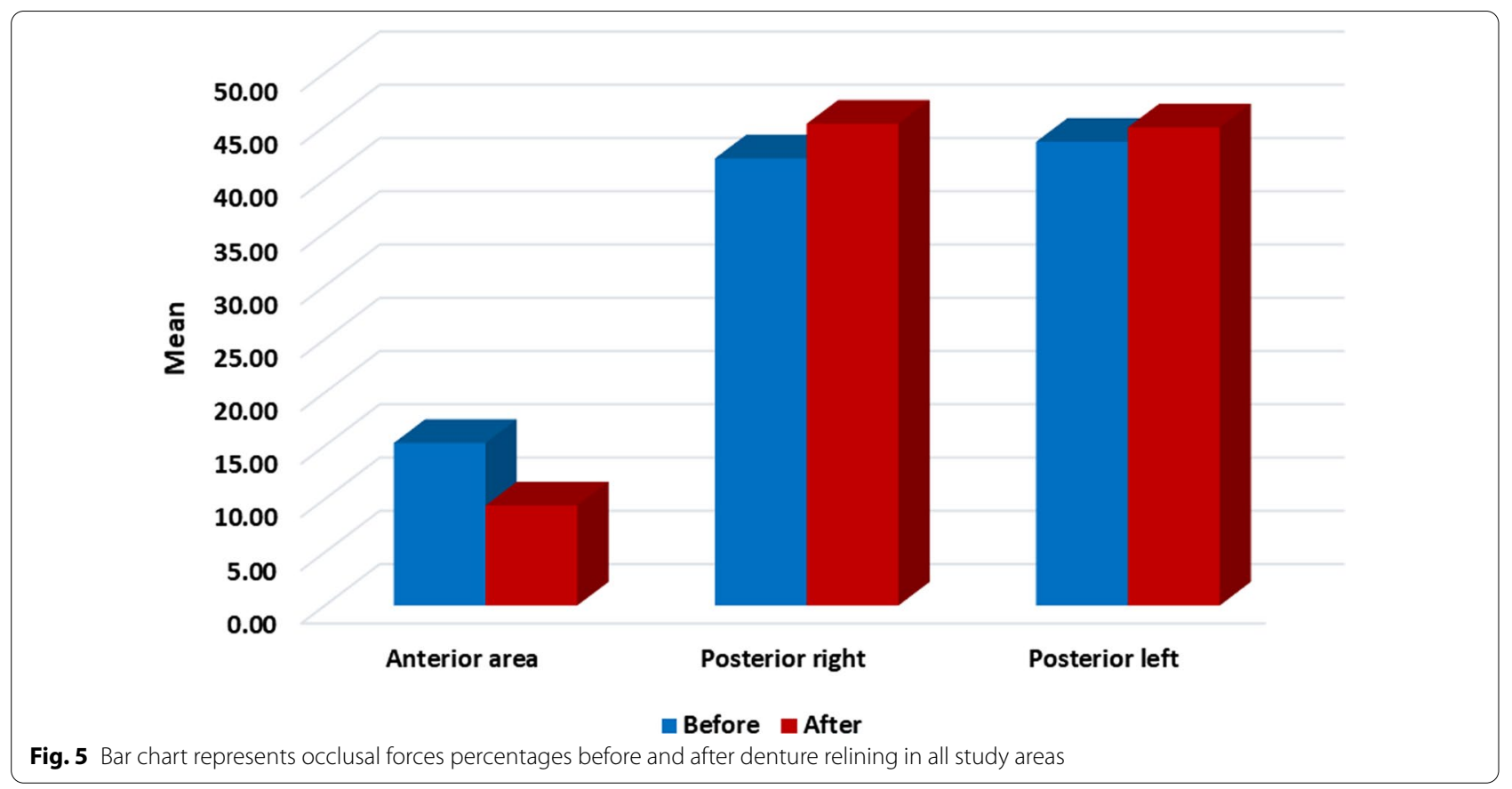

using articulating paper due to mucosal resiliency. Thus, leading to better force distribution patterns on the right and left sides simultaneously after relining and moreover decreasing the anterior share of occlusal load which implies that, the articulating paper may be not sufficient to obtain bilateral occlusal balance in complete dentures.

Furthermore, the right side showed significantly higher percentage of occlusal force after relining which may be due to the role of adhesive in reducing possible displacement and dislodgement of the denture during function, aiding in better fitness, adaptation and also reducing the rotational movement of the denture and accordingly the time needed for food mastication (Maruo et al. 2010).

\section{Conclusions}

Within the limitations of the study design, it could be concluded that using digital occlusal analysis (T-scan) and proper denture relining significantly improved the force distribution in initially ill-fitting complete dentures, and further enhanced denture retention/stability ensuring simultaneous occlusal contacts.

\section{Abbreviations}

PMMA: Poly-methyl methacrylate; LTSL: Long-term soft lining material.

\section{Acknowledgements}

None.

\section{Authors' contributions}

E.I. has made the study design and data analysis. H.E. has made data interpretation and revised the research article. All authors have read and approved the manuscript.

\section{Funding}

This research is a part of 3-years project (2016-2019) funded and supported by National Research Centre (Project ID: 11010203) Cairo, Egypt.

\section{Availability of data and materials}

The datasets used and/or analyzed during the current study are available from the corresponding author on reasonable request.

\section{Declarations}

Ethic approval and consent to participate

This study was designed and approved by the Medical Research Ethical committee (MREC Approval No. 16086) of National Research Centre (NRC), Cairo-Egypt, which is in accordance with Helsinki Declaration of 1975. All patients were informed about the practical steps of this study and signed written approval consent.

\section{Consent for publication}

Not applicable.

\section{Competing interests}

The authors declare that they have no competing interests.

\section{Author details}

${ }_{1}^{1}$ Prosthodontics Department, National Research Centre, Cairo, Egypt. ${ }^{2}$ Prosthodontics Department, National Research Centre-MSA University, Cairo, Egypt.

Received: 17 February 2021 Accepted: 11 April 2021

Published online: 19 May 2021

\section{References \\ Atay A, Cetintas VB, Cal E, Kosova B, Kesercioglu A, Guneri P (2012) Cytotoxicity of hard and soft denture lining materials. Dent Mater J 31(6):1082-1086 Doundoulakis JH, Eckert SE, Lindquist CC, Jeffcoat MK (2003) The implant- supported overdenture as an alternative to the complete mandibular denture. J Am Dent Assoc 134:1455-1458}


Elmorsy AE, Ibraheem EM, Aboul Ela A, Fahmy A, Nassani MZ (2015) Do flexible acrylic resin lingual flanges improve retention of mandibular complete dentures? J Int Soc Prev Community Dent 5(5):365-371

Gümüs HÖ, Kılınç HI, Tuna SH, Ozcan N (2013) Computerized analysis of occlusal contacts in bruxism patients treated with occlusal splint therapy. J Adv Prosthodont 5:256-261

Ibraheem EM, Hammad HG (2019) Effect of commercially available denture adhesives on microhardness of a flexible denture base material. Open Access Macedonian J Med Sci 7(5):862-868

Ibraheem EM, Hammd HG (2020) Impact of long-term soft relining of mandibular complete dentures on brain activity and cognitive function of elderly patients. Open Access Maced J Med Sci 8(D):158-165

Ibraheem EM, Hammad HG, El-Sisy AM (2019) Comparing marginal bone height changes around immediately and delayed implant-retained mandibular overdentures in controlled diabetic patients: a randomized clinical study. Bull Natl Res Centre 43:198

Jain P, Rathee M (2019) Stability in mandibular denture. Copyright @ 2020, Stat Pearls Publishing LLC. Bookshelf ID: NBK549861, PMID 3175105

Kansal G, Goyal D (2013) Prosthodontic management of patients with diabetes mellitus. J Adv Med Dent Scie Res 1(1):38-44

Kerstein RB (2008) Articulating paper mark misconceptions and computerized occlusal analysis technology. Dent Implantol Update 19:41-46

Kerstein RB, Radke J (2006) The effect of disclusion time reduction on maximal clench muscle activity levels. Cranio 24:156-165

Kerstein RB, Grundset K (2001) Obtaining bilateral simultaneous occlusal contacts with computer analyzed and guided occlusal adjustments. Quintessence Int 32:7-18

Kerstein RB, Thumati P, Padmaja S (2013) Force finishing and centering to balance a removable complete denture prosthesis using the T- Scan III computerized occlusal analysis system. J Indian Prosthodont Soc 13:184-188

Koos B, Godt A, Schille C, Göz G (2010) Precision of an instrumentation-based method of analysing occlusion and its resulting distribution of forces in the dental arch. J Orofac Orthop 71:403-410

Kreve S, Dos Reis A (2019) Denture liners: a systematic review relative to adhesion and mechanical properties. Sci World J Article ID 6913080

Kuć J, Sierpińska T, Gołębiewska M (2017) Evaluation of functional parameters in the occlusion of complete denture wearers before and after prosthetic treatment. J Prosthodont Res 61(4):480-490
Maness WL, Benjamin M, Podoloff R, Bobick A, Golden RF (1987) Computerized occlusal analysis: a new technology. Quintessence Int 18:287-292

Martins MJS, Caramelo FJ, Ramalho da Fonseca JA, Nicolau PMG (2014) In vitro study on the sensibility and reproducibility of the new T-Scan III HD system. Rev Port Estomatol Med Dent Cir Maxilofac 55:14-22

Maruo Y, Nishigawa G, Irie M, Oka M, Hara T, Suzuki K et al (2010) Stress distribution prevents ischaemia and bone resorption in residual ridge. Arch Oral Biol 55:873-878

Mauri-Obradors E, Estrugo-Devesa A, Jané-Salas E, Viñas M, LópezLópez J (2017) Oral manifestations of diabetes mellitus. A systematic review. Med Oral Patol Oral Cir Bucal 22(5):e586-e594

Metwally AF (2019) Comparison of patient satisfaction and occlusal force distribution pattern in CAD/CAM and conventional complete dentures using the T-Scan III computerized occlusal analysis system (RCT). Egypt Dent J 65(3):2641-2649

Misch CE (2008) Contemporary implant dentistry. Elsevier Mosby, pp 15, 134-141, 294-299, 300-308, 421-466, 654, 812-813, 818, 833, 1073

Pradeep N, Devassy JP, Sreekumar AV (2015) Sublingual crescent extension: a solution for loose lower denture. Case Report Saint Int Dent J 1(1):66-72

Qadeer S, Kerstein R, Kim RJY, Huh JB, Shin SW (2012) Relationship between articulation paper mark size and percentage of force measured with computerized occlusal analysis. J Adv Prosthodont 47:7-12

Rahman B (2013) Prosthodontic concerns in a diabetic patient. Int J Health Sci Res 3(10):117-120

Rani I, Shetty J, Reddy V (2017) A comparison of peri-implant strain generated by different types of implant supported prostheses. J Indian Prosthodont Soc 17:142-148

Soman B, Bhatnagar S (2014) Adverse effects of drugs on salivary glands. Int J Dent Med Res 1(4):94-98

Tallgren A, Lang BR, Holden S, Huyser DJ, Miller RL (1992) Longitudinal electromyographic study of chewing patterns in complete denture wearers. Int J Prosthodontics 5:415

World Health Organization (2019) PDF, p 14

\section{Publisher's Note}

Springer Nature remains neutral with regard to jurisdictional claims in published maps and institutional affiliations.

\section{Submit your manuscript to a SpringerOpen ${ }^{\circ}$ journal and benefit from:}

- Convenient online submission

- Rigorous peer review

- Open access: articles freely available online

- High visibility within the field

Retaining the copyright to your article

Submit your next manuscript at springeropen.com 